\title{
GENERALIZED SUPER-PARABOLIC FUNCTIONS
}

\author{
BY NEIL EKLUND
}

Communicated by Alberto Calderón, September 22, 1973

The purpose of this note is to announce results which generalize potential theory (superharmonic functions) to a broad class of parabolic operators. Many of the properties of superharmonic functions carry over to functions in this new class. Let $Q=\Omega \times(0, T)$ where $\Omega \subset E^{n}$ is a bounded domain and $T>0$ is a scalar. All functions will be defined on $\bar{Q}$ and will be written as functions of $(x, t)$ with $x \in \bar{\Omega}$ and $t \in[0, T]$.

For $(x, t) \in \bar{Q}$ assume

(a) $a_{i j}(x, t)$ is a bounded, measurable function for $i, j=1,2, \cdots, n$ and assume there is a constant $\lambda>0$ such that $\sum a_{i j}(x, t) z_{i} z_{j} \geqq \lambda|z|^{2}$ for all $z \in E^{n}$ and almost all $(x, t) \in Q$.

(b) $c(x, t) \in L^{q}\left[0, T ; L^{p}(\Omega)\right]$ for $n / 2 p+1 / q<1,1<p, q \leqq \infty$.

(c) $b_{j}(x, t), d_{j}(x, t) \in L^{q}\left[0, T ; L^{p}(\Omega)\right]$ for $j=1, \cdots, n$ and $n / 2 p+1 / q<\frac{1}{2}$, $2<p, q \leqq \infty$.

The parabolic operator under consideration is defined by

$$
L u=u_{t}-\left\{a_{i j}(x, t) u_{, i}+d_{j}(x, t) u\right\}_{, j}-b_{j}(x, t) u_{, j}-c(x, t) u
$$

where $u_{, j}=\partial u / \partial x_{j}$ and an index $i$ or $j$ is summed over $1 \leqq i, j \leqq n$ whenever it is repeated in a product.

DEFINITION 1. $u(x, t)$ is a weak solution of $L u=0$ in $Q$ if $u$ is locally in $L^{2}\left[0, T ; H^{1,2}(\Omega)\right]$ and $\iint_{Q}\left[a_{i j} u, \phi_{, j}+d_{j} \phi_{, j} u-b_{j} u_{, j} \phi-c u \phi-u \phi_{t}\right] d x d t=$ 0 for all $\phi \in C_{0}^{1}(Q)$.

Let $\partial_{p} Q=\{\partial \Omega \times[0, T]\} \cup\{\Omega \times(0)\}$ denote the parabolic boundary of $Q$. Due to the number of definitions and results, they are stated below with no proofs.

THEOREM 1. Let $f \in C\left(\partial_{p} Q\right)$ and let $u=u(x, t)$ be the weak solution of the boundary value problem

$$
L u=0 \quad \text { on } Q . \quad u=f \text { on } \partial_{p} Q .
$$

Then, to each $(x, t) \in Q$, there corresponds a nonnegative Borel measure

AMS (MOS) subject classifications (1970). Primary 35K20, 31C05; Secondary 35D05. Key words and phrases. Superharmonic functions, parabolic operators. 
$\mu_{(x, t)}$ on $\partial_{p} Q$ such that

$$
u(x, t)=\int_{\partial_{p} Q} f d \mu_{(x, t)} \text { on } Q .
$$

In the future, write $L(f ;(x, t), Q)=\int_{\partial_{p} Q} f d \mu_{(x, t)}$.

DEFINITION 2. $u \in S_{Q}$ if and only if $u \in L^{2}\left[0, T ; H^{1,2}(\Omega)\right]$ and for all $\phi \in C_{0}^{1}\left(Q^{i}\right)$ with $\phi \geqq 0, \iint_{Q}\left[a_{i j} u, \phi_{, j}+d_{j} \phi_{, j} u-b_{j} u_{, j} \phi-c u \phi-u \phi_{t}\right] d x d t \geqq 0$.

Definition 3. $R_{a}\left(x_{0}, t_{0}\right) \equiv\left\{(x, t) ;\left|x_{i}-x_{0 i}\right|<a, t_{0}-a^{2}<t \leqq t_{0}\right\}$ is called a standard rectangle based at $\left(x_{0}, t_{6}\right)$.

DEFINITION 4. $u \in l(Q)$ if and only if

(i) $u \neq \equiv+\infty$ on $Q$,

(ii) $u>-\infty$ on $Q$, and

(iii) $u$ is lower semicontinuous on $Q$.

Definition 5. The extended real valued Borel measurable function $u$ defined on an open set $D$ is

(a) super-mean-valued at $z \in D$ if $L\left(u ; z, R_{\delta}\right)$ is defined and $u(z) \geqq$ $L\left(u ; z, R_{\delta}\right)$ for almost all $\delta$ with $\bar{R}_{\delta} \subset D$;

(b) super-mean-valued on $D$ if it is super-mean-valued at each $z \in D$;

(c) locally super-mean-valued at $z \in D$ if there is a $\delta(z)>0$ such that $\bar{R}_{\delta(z)} \subset D$ and $u(z) \geqq L\left(u ; z, R_{\delta}\right)$ for all $\delta<\delta(z)$;

(d) locally super-mean-valued on $D$ if it is locally super-mean-valued at each $z \in D$.

Definition 6. $S_{Q}^{\prime}=\{u \in l(Q) ; u$ is super-mean-valued on $Q\}$. $S_{Q}^{\prime \prime}=$ $\{u \in l(Q)$; for any cylinder $W=C \times(a, b)$ with $\bar{W} \subset Q$, and any $v$ with $v \in C(\bar{W}), L v=0$ on $W$, and $u \geqq v$ on $\partial_{p} W$, it follows that $u \geqq v$ on $\left.W\right\}$. $S^{\prime \prime \prime}=\{u \in l(Q) ; u$ is locally super-mean-valued on $D\}$.

THEOREM 2. $u \in S_{Q}$ with $u \geqq 0$ on $\partial_{p} Q$ implies $u \geqq 0$ on $Q$.

COROLlaRY. If $c+\left\{d_{j}\right\}_{, j} \leqq 0$ weakly on $Q$, then the weak solution $u$ of $L u=0$ in $Q, u=1$ on $\partial_{p} Q$ satisfies $0 \leqq u(x, t) \leqq 1$ on $Q$.

From now on assume $c+\left\{d_{j}\right\}_{, j} \leqq 0$ weakly on $Q$.

THEOREM 3. Let $u \in S_{\mathrm{Q}}^{\prime \prime \prime}$. If, for some $\left(x_{0}, t_{0}\right) \in Q, u\left(x_{0}, t_{0}\right)=\inf _{Q} u \leqq 0$, then $u(x, t) \equiv u\left(x_{0}, t_{0}\right)$ on $\Omega \times\left(0, t_{0}\right)$.

THEOREM 4. $S_{Q} \subset S_{Q}^{\prime}=S_{Q}^{\prime \prime}=S_{Q}^{\prime \prime \prime}$.

THEOREM 5. Let $F(x)$ be convex on $E^{n}$ with $F(0) \leqq 0$. If $L u=0$ on $Q$, then $-F(u) \in S_{Q}^{\prime}$.

THEOREM 6. Let $F(x)$ be nondecreasing and convex on $E^{n}$ with $F(0) \leqq 0$. If $-u \in S_{Q}^{\prime}$, then $-F(u) \in S_{Q}^{\prime}$. 
THEOREM 7. If $u \in S_{Q}^{\prime}$, and if $u(x, t) \geqq 0$, then there exist $t_{0}$, $t_{1}$ with $0 \leqq t_{0} \leqq t_{1} \leqq T$ such that

$$
\begin{array}{ll}
u(x, t) \equiv 0 & \text { on } \Omega \times\left(0, t_{0}\right) \\
0<u(x, t)<+\infty & \text { on } \Omega \times\left(t_{0}, t_{1}\right), \\
u(x, t) \equiv+\infty & \text { on } \Omega \times\left(t_{1}, T\right)
\end{array}
$$

THEOREM 8. If $u, v \in S_{Q}^{\prime}$ and $c>0$, then (i) $c u \in S_{Q}^{\prime}$, (ii) $u+v \in S_{Q}^{\prime}$, (iii) $\inf (u, v) \in S_{Q}^{\prime}$.

THEOREM 9. $u,-u \in S_{Q}^{\prime}$ implies $L u=0$ weakly on $Q$. Set

THEOREM 10. Let $u \in S_{Q}^{\prime}$ and let $R$ be a standard rectangle with $\bar{R} \subset Q$.

$$
\begin{aligned}
v(x, t) & =L(u ;(x, t), R) & & (x, t) \in R, \\
& =u(x, t) & & (x, t) \in Q-R .
\end{aligned}
$$

Then $u \geqq v$ on $Q, L v=0$ on $R$, and $v \in S_{Q}^{\prime}$.

\section{REFERENCES}

1. D. G. Aronson, Non-negative solutions of linear parabolic equations, Scuola Norm. Sup. Pisa 22 (1968), No. 4, 607-694.

2. D. G. Aronson and James Serrin, Local behavior of solutions of quasilinear parabolic equations, Arch. Rational Mech. Anal. 25 (1967), 81-122. MR 39 \#5952.

3. Neil Eklund, Boundary behavior of solutions of parabolic equations with discontinuous coefficients, Bull. Amer. Math. Soc. 77 (1971), 788-792. MR 45 \#2311.

4. Avner Friedman, Partial differential equations of parabolic type, Prentice-Hall, Englewood Cliffs, N.J. 1964. MR 31 \#6062.

5. O. A. Ladyženskaja and N. N. Ural'ceva, Linear and quasilinear elliptic equations, "Nauka", Moscow, 1964; English transl., Academic Press, New York, 1968. MR 35 \#1955; 39 \#5941.

6. W. Littman, G. Stampacchia and H. Weinberger, Regular points for elliptic equations with discontinuous coefficients, Ann. Scuola Norm. Sup. Pisa (3) 17 (1963), 43-77. MR 28 \#4228.

7. Walter Rudin, Real and complex analysis, McGraw-Hill, New York, 1966. MR 35 \#1420.

8. Neil Trudinger, Pointwise estimates and quasilinear parabolic equations, Comm. Pure Appl. Math. 21 (1968), 205-226. MR 37 \#1758.

Department of Mathematics, Vanderbilt University, Nashville, Tennessee 37235 